\title{
CONNEXIONS IN DIFFERENTIAL GEOMETRY OF HIGHER ORDER
}

\author{
BY \\ WILLIAM F. POHL( $\left.{ }^{1}\right)$
}

1. In a previous paper [10, hereafter cited DGHO] the author studied the osculating spaces of submanifolds of affine and projective spaces. This led to a theory of affine and projective singularities (e.g., inflection points, planar points) which were described by the falling-in-rank of certain vector-bundle homomorphisms. This theory he then developed in the complex-analytic case and obtained generalizations of the Plücker formulas of algebraic geometry. In his thesis E. A. Feldman [5] developed the theory extensively for real differentiable manifolds and obtained transversality theorems and theorems on the nonexistence of singularity-free immersions. These theorems constitute nearly all that is known about real affine differential geometry in-the-large in higher dimensions.

The main purpose of the present paper is to generalize the theory to submanifolds of affinely connected spaces, and following a suggestion of S. S. Chern, to submanifolds of projectively connected spaces. The main features of the affine case remain the same. To treat the projective case we shall show that every projective connexion has an underlying affine connexion and that the osculating spaces and inflection points defined with respect to this affine connexion depend only on the projective connexion. The geometry of spaces with projective connexion seems to be the natural setting for many of the main results of Feldman's thesis.

In reality the generalization to affinely connected spaces has already been carried out by Feldman. He has developed the theory for a space with affine connexion together with a further structure which he calls a sequence of dissections or " $p$ th order symmetric linear connexions." Our essential contribution is to show that the affine connexion already determines the additional structure in a natural way. As an example of the theory we shall state and prove one of Feldman's theorems.

The main results of this paper are two theorems on connexions. Let $M$ be a differentiable manifold and let $T_{p}$ be the $p$ th order osculating bundle of $M$, i.e., the bundle of linear differential operators of order $\leqq p$ on functions on $M$. In DGHO the author established a natural exact sequence

$$
\mathfrak{T}_{p}(M): 0 \rightarrow T_{p-1} \rightarrow T_{p} \rightarrow \bigcirc^{p} T_{1} \rightarrow 0
$$

where $\bigcirc$ denotes the symmetric tensor product. A pth order dissection in the sense

Received by the editors January 28, 1964 and, in revised form, April 28, 1966.

( $\left.{ }^{1}\right)$ Research supported by the National Science Foundation under grant 20301. 
of Feldman is essentially a splitting of this exact sequence. We shall show that a connexion in $T_{1}$, i.e., an affine connexion on $M$, induces a $p$ th order dissection and a connexion in $T_{p}$, for each $p$, and that these structures are characterized in a natural geometrical way.

In $\S 3$ we show that a connexion in an arbitrary vector bundle $V$ on $M$, together with an affine connexion in $M$, induce connexions in the derived bundles $\Delta_{p} V$ and splittings of various exact sequences involving these derivatives. These considerations lead to a new interpretation of the curvature. In the Appendix we discuss some alternative definitions of these derivatives which have been pointed out by D. C. Spencer, N. H. Kuiper, and P. Libermann.

The author wishes to thank E. A. Feldman for numerous and fruitful conversations on these questions and for pointing out an error in an earlier version of this paper. He also wishes to thank H. Samelson for stimulating discussions of these problems.

2. For our purposes we need a new definition of the notion of a connexion in a vector bundle.

We shall use the conventions of notation and terminology of DGHO throughout, so that "manifold," "map," etc. may be interpreted either as " $C^{\infty}$ differentiable manifold," " $C^{\infty}$ map," etc., or as "complex-analytic manifold," "complex-analytic map," etc. However, the main interest of our results is in the real case.

Let $V \rightarrow M$ be a vector bundle over a manifold $M$, and let $T_{1}$ denote the tangent bundle of $M$. In DGHO the author defined a new vector bundle over $M, \Delta_{1} V$, called "the first derivative of $V$," and established an exact sequence

$$
\mho_{1}(V): 0 \longrightarrow V \stackrel{I_{V}}{\longrightarrow} \Delta_{1} V \stackrel{P_{V}}{\longrightarrow} T_{1} \otimes V \longrightarrow 0 .
$$

Definition. A splitting of $\aleph_{1}(V)$ will be called a connexion in $V$.

A splitting of an exact sequence $0 \rightarrow A \rightarrow{ }^{f} B \rightarrow{ }^{g} C \rightarrow 0$ gives rise to maps $\pi: B \rightarrow A$ and $\tilde{\pi}: C \rightarrow B$ satisfying the conditions $\pi f=\mathrm{id}$. and $g \tilde{\pi}=\mathrm{id}$., and conversely a map $\pi$ or $\tilde{\pi}$ satisfying the respective condition determines a splitting. It will be convenient for our purposes to call $\pi$ itself the splitting. We shall call $\tilde{\pi}$ the map associated to $\pi$.

Proposition 1. The definition of "connexion" above is equivalent to the standard definitions.

Proof. Let $\mathscr{V}$ be the sheaf of germs of sections of $V, \mathscr{T}_{1}$ the sheaf of germs of tangent vector fields, $\mathscr{D}$ the sheaf of germs of differentiable (or holomorphic) functions, and $\mathscr{C}$ the sheaf of germs of constant functions. Recall that $\Delta_{1} \mathscr{V}=\mathscr{V} \oplus \mathscr{T}_{1} \otimes_{\mathscr{C}} \mathscr{V} \mid \mathscr{R}$, where $\mathscr{R}$ is the $\mathscr{D}$-subsheaf generated by all elements of the form $-D(f) b \oplus(D \otimes f b-f D \otimes b)$. Let $q: \mathscr{V} \oplus \mathscr{T}_{1} \otimes_{\mathscr{C}} \mathscr{V} \rightarrow \Delta_{1} \mathscr{V}$ be 
the canonical projection. $I_{V}(a)=q(a \oplus 0)$, so a $\mathscr{D}$-homomorphism $\pi: \Delta_{1} \mathscr{V} \rightarrow \mathscr{V}$ is a splitting of $\mho_{1}(\mathscr{V})$ if and only if $\pi q(a \oplus 0)=a$. Hence to give a splitting of $\mathfrak{E}_{1}(\mathscr{V})$ is to give a map $\nabla: \mathscr{T}_{1} \otimes_{\mathscr{B}} \mathscr{V} \rightarrow \mathscr{V}$ subject to the conditions

1. $\nabla_{X+X}, b=\nabla_{X} b+\nabla_{X}, b$,

2. $\nabla_{f X} b=f \nabla_{X} b$,

3. $\nabla_{X}\left(b+b^{\prime}\right)=\nabla_{X} b+\nabla_{X} b^{\prime}$,

4. $\nabla_{X} f b=X(f) b+f \nabla_{X} b$

(where we have written $\nabla_{X} b$ for $\nabla(X \otimes b)$ ). These are the basic properties of the covariant derivative for a connexion in a vector bundle [8, pp. 113-116].

Suppose given a map $\nabla$ as above. Let $U$ be an open set of $M$ over which there are $n$ sections $S^{1}, \ldots, S^{n}$ of $V$, which span the fibre and are linearly independent at each point. We define a matrix-valued one-form in $U$ by the equation $\sum \theta_{i}^{j}(X) S^{i}=\nabla_{X} S^{j}$. If $W$ is an open set with sections $T^{1}, \ldots, T^{n}$ as above, with the transition law $S^{i}=\sum f_{U W j}^{i} T^{j}$, and $\theta_{i}^{\prime j}$ the one-form defined as above with respect to the $T$ 's, we derive the law of transition

$$
\theta_{j}^{\prime k}(X)=\sum_{l} X\left(f_{W U l}^{k}\right) f_{U W_{j}^{j}}^{l}+\sum_{l, m} f_{W U l}^{k} \theta_{m}^{l}(X) f_{U W j}^{m}
$$

which we may rewrite as

$$
\sum f_{W U i}^{j} \theta_{j}^{\prime k}=d f_{W U i}^{k}+\sum f_{W U l}^{k} \theta_{i}^{l} .
$$

But to give such a collection of forms subject to this transformation law is just to give a connexion (in the standard sense) in the bundle $V$, according to Chern [3].

Conversely, given a collection of forms as above, we may define $\nabla$ by

$$
\nabla_{X}\left(\sum f_{i} S^{i}\right)=\sum\left(X f_{i}\right) S^{i}+\sum \theta_{j}^{i}(X) S^{j}
$$

It is easy to check that $\nabla_{X}$ is independent of the coordinate system for $V$ and satisfies the properties 1-4 above. This completes the proof of Proposition 1.

In this connection we seek a new expression for the curvature, which according to Chern [3], is a collection of matrix-valued two-forms in local coordinates defined by

$$
\Theta_{U j}^{i}=d \theta_{j}^{i}+\sum_{k} \theta_{j}^{k} \wedge \theta_{k}^{i}
$$

and satisfying the transformation law $\Theta_{U}=\operatorname{ad}\left(g_{U W}\right) \Theta_{w}$, which is to say that they constitute a globally defined two-form with values in the bundle of Lie algebras, End $V$. By standard formulas [11],

$$
\begin{aligned}
\sum \Theta_{U}^{i}(X \wedge Y) S^{j} & =\sum\left\{d \theta_{j}^{i}(X \wedge Y)+\sum \theta_{j}^{k} \wedge \theta_{k}^{i}(X \wedge Y)\right\} S^{j} \\
& =\sum_{j, k}\left\{-\theta_{j}^{i}([X, Y])+X\left(\theta_{j}^{i}(Y)\right)-Y\left(\theta_{j}^{i}(X)\right)+\left|\begin{array}{ll}
\theta_{j}^{k}(X) & \theta_{j}^{k}(Y) \\
\theta_{k}^{i}(X) & \theta_{k}^{i}(Y)
\end{array}\right|\right\} S^{j} \\
& =-\nabla_{[X, Y]} S^{i}+\nabla_{X} \nabla_{Y} S^{i}-\nabla_{Y} \nabla_{X} S^{i} .
\end{aligned}
$$


3. Suppose given a commutative diagram

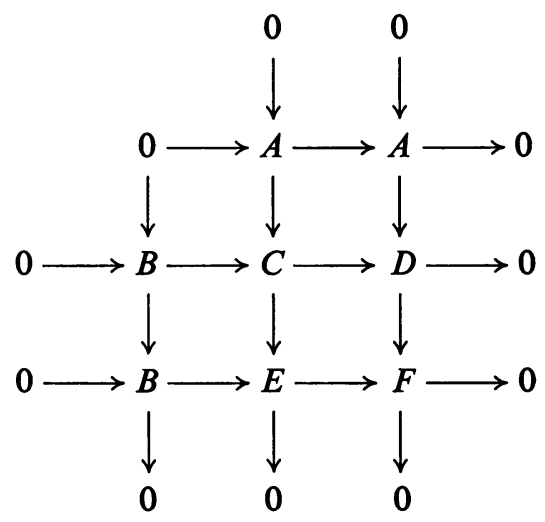

with exact rows and columns. Let $\tilde{\omega}: C \rightarrow B$ be a splitting of the middle row. Then we call the composition $A \rightarrow C \rightarrow B$, which is an element in $\operatorname{Hom}(A, B)$, the obstruction to splitting the bottom row.

LEMMA 2. 1. A splitting $o: E \rightarrow B$ of the bottom row of I gives rise to a splitting $\pi: C \rightarrow E \rightarrow B$ of the middle row of I with obstruction equal to zero.

2. A splitting of the middle row of I with associated map $D \rightarrow C$, and a splitting of the right-hand column with associated map $F \rightarrow D$ gives rise to a splitting of the bottom row with associated map $F \rightarrow D \rightarrow C \rightarrow E$.

3. A splitting of the middle row of I with obstruction equal to zero induces a splitting of the bottom row.

Proof. Elementary diagram chasing.

Let $V$ be a vector bundle over a manifold $M$. Recall that in DGHO the author defined new vector bundles $\Delta_{p} V$ and $\tilde{\Delta}_{p} V$, for each positive integer $p$. These were related by the commutative diagram with exact rows and columns

II

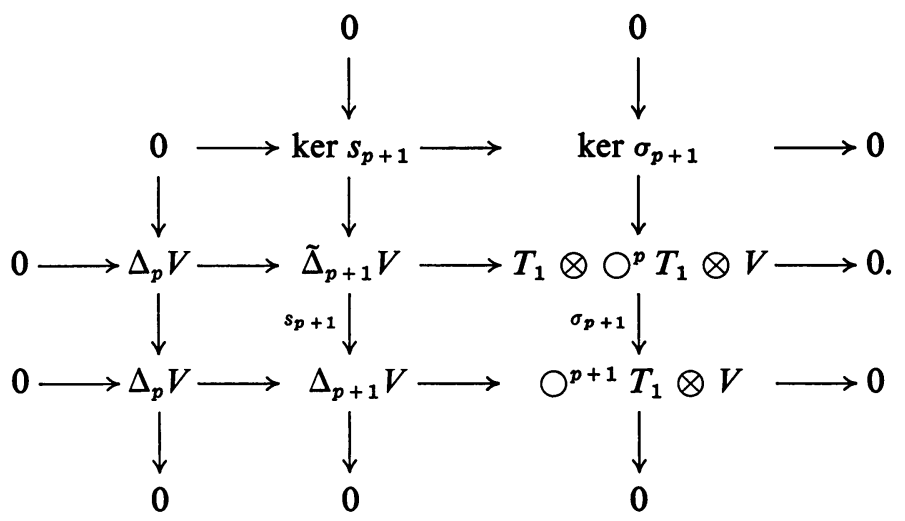

If $p=1$, the kernel of $\sigma_{2}$ is $T_{1} \wedge T_{1} \otimes V$, and diagram II may be changed to the commutative diagram with exact rows and columns. 
III

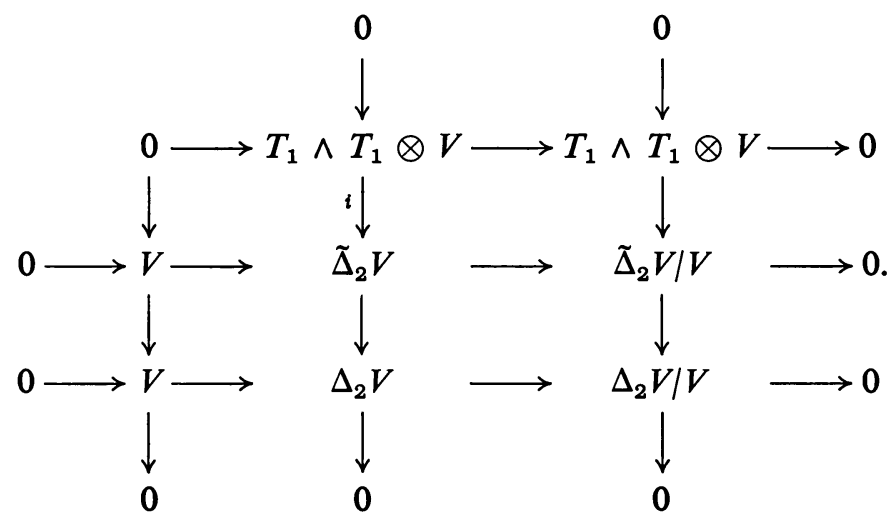

Now let $\tilde{\omega}: \Delta_{1} V \rightarrow V$ be a connexion in $V$. In DGHO it is shown that the product connexion in the product bundle $\tilde{\omega}_{1}: \Delta_{1} I^{n} \rightarrow I^{n}$ induces splittings $\tilde{\omega}_{p}^{\prime}: \tilde{\Delta}_{p} I^{n} \rightarrow I^{n}$. The construction was recursive and for $p=2$ did not depend on any property peculiar to the product connexion. The argument shows that the connexion $\tilde{\omega}: \Delta_{1} V \rightarrow V$ induces a splitting $\tilde{\omega}_{2}^{\prime}: \tilde{\Delta}_{2} V \rightarrow V$, of the middle row of III.

Proposition 3. The obstruction of $\tilde{\omega}_{2}^{\prime}$, in diagram III, which is a cross-section of Hom $\left(T_{1} \wedge T_{1} \otimes V, V\right)=T_{1}^{*} \wedge T_{1}^{*} \otimes L(V)$, i.e., a two-form with values in the bundle of Lie algebras associated to $V$, is the curvature of the connexion $\tilde{\omega}$.

Proof. The map $i: T_{1} \wedge T_{1} \otimes V \rightarrow \tilde{\Delta}_{2} V$ of diagram III is given by $i(E \wedge F \otimes e)=r_{2} q(q \oplus$ id. $\otimes q)[0 \oplus E \otimes(0 \oplus F \otimes e)$

$$
-0 \oplus F \otimes(0 \oplus E \otimes e)-0 \oplus[E, F] \otimes(e \oplus 0)] \text {. }
$$

(cf. DGHO, p. 186). Hence, by an elementary computation,

$$
\tilde{\omega}_{2}^{\prime} i[E \wedge F \otimes e)=\left(\nabla_{E} \nabla_{F}-\nabla_{F} \nabla_{E}-\nabla_{[E, F]}\right)(e) \text {, }
$$

which is the curvature, by the formula at the end of the previous section. Q.E.D.

The right-hand column of III may be embedded in the commutative diagram with exact rows and columns

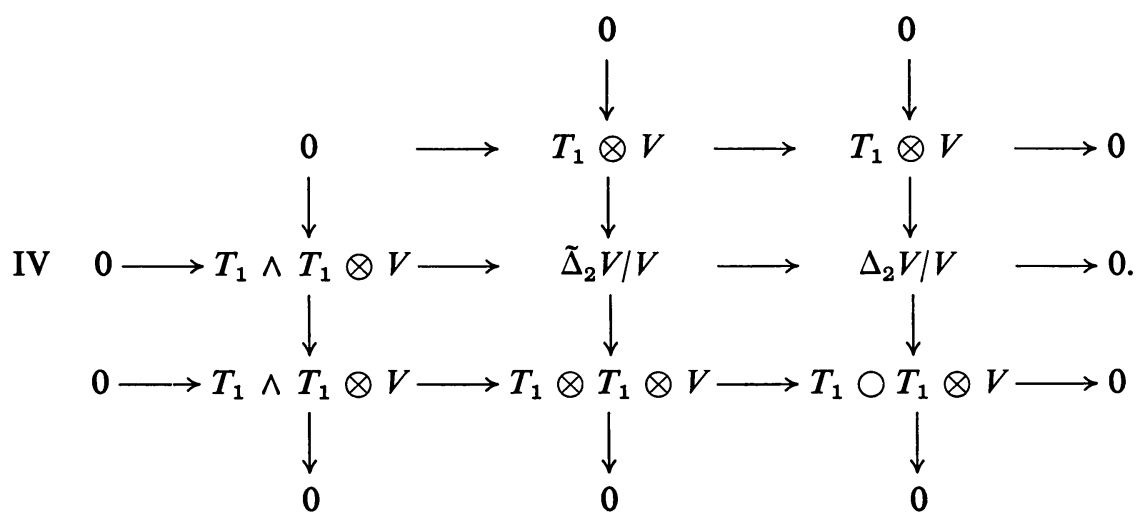


The bottom row of IV has a natural splitting induced by the alternation map, which induces a splitting of its middle row. This splitting, together with $\tilde{\omega}_{2}^{\prime}$, induces a splitting of the bottom row of III, by Lemma 2 . But if one attempts to continue the process to find a map $\tilde{\omega}_{3}^{\prime}: \tilde{\Delta}_{3} V \rightarrow V$ he encounters the curvature as an obstruction, as may be seen from the computation at the top of p. 193 of DGHO.

Proposition 4. $A$ connexion in $V$, together with a connexion in $T_{1}$, induces $a$ connexion $\xi_{p}$ in $\Delta_{p} V$ and a splitting $o_{p+1}$ of the exact sequence

$$
\circledast_{p+1}(V): 0 \rightarrow \Delta_{p} V \rightarrow \Delta_{p+1} V \rightarrow \bigcirc^{p+1} T_{1} \otimes V \rightarrow 0,
$$

for each $p \geqq 0$.

Proof. We proceed by induction. Let $\xi_{0}: \Delta_{1} \Delta_{0} V=\Delta_{1} V \rightarrow V$ be the given connexion, and let $o_{0}=\mathrm{id} .: \Delta_{0} V=V \rightarrow V$. Now suppose that $\xi_{p}$ and $o_{p}$ are already defined. Then the map associated to $o_{p}, \tilde{o}_{p}$ gives rise to a splitting

$$
\bigcirc^{p+1} T_{1} \otimes V \rightarrow \otimes^{p+1} T_{1} \otimes V \rightarrow T_{1} \otimes \bigcirc^{p} T_{1} \otimes V \stackrel{\text { id. } \otimes \tilde{o}_{p}}{\longrightarrow} T_{1} \otimes \Delta_{p} V
$$

of the right-hand column of the commutative diagram with exact rows and columns

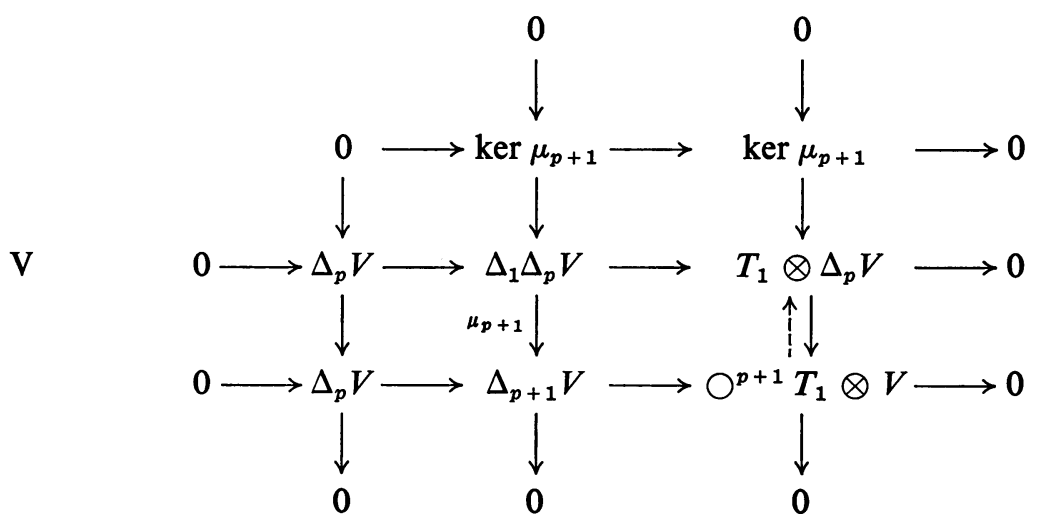

(cf. DGHO, p. 186, for definition of $\mu_{p+1}$ ). This splitting, together with the connexion $\xi_{p}$, gives rise to a splitting $o_{p+1}: \Delta_{p+1} V \rightarrow \Delta_{p} V$ of the bottom row of $V$, which gives rise to an isomorphism $\Delta_{p+1} V \cong \Delta_{p} V \oplus \bigcirc^{p+1} T_{1} \otimes V$. But $\bigcirc^{p+1} T_{1}$ is algebraically associated to $T_{1}$, and therefore has a connexion induced from the given one. And a connexion in each of two bundles induces connexions in their direct sum and tensor product according to Chern [3]. Therefore $\xi_{p}$ and the given connexions induce a connexion $\xi_{p+1}$ in $\Delta_{p+1} V$. This completes the proof.

4. In studying the relations between the osculating bundles of higher order and the various derivatives of the tangent bundle of a manifold $M$ the author 
defined a vector-bundle homomorphism $\varepsilon_{p}$ of $\Delta_{1} T_{p-1}$ onto $T_{p}$ (cf. DGHO, Proposition 3.20), and showed, furthermore, that the $\varepsilon$ 's give rise to a mapping of exact sequences $\mathbb{E}_{1}\left(T_{p-1}\right) \rightarrow \mathfrak{T}_{p}$. For $p=2$ this gives the commutative diagram with exact rows and columns

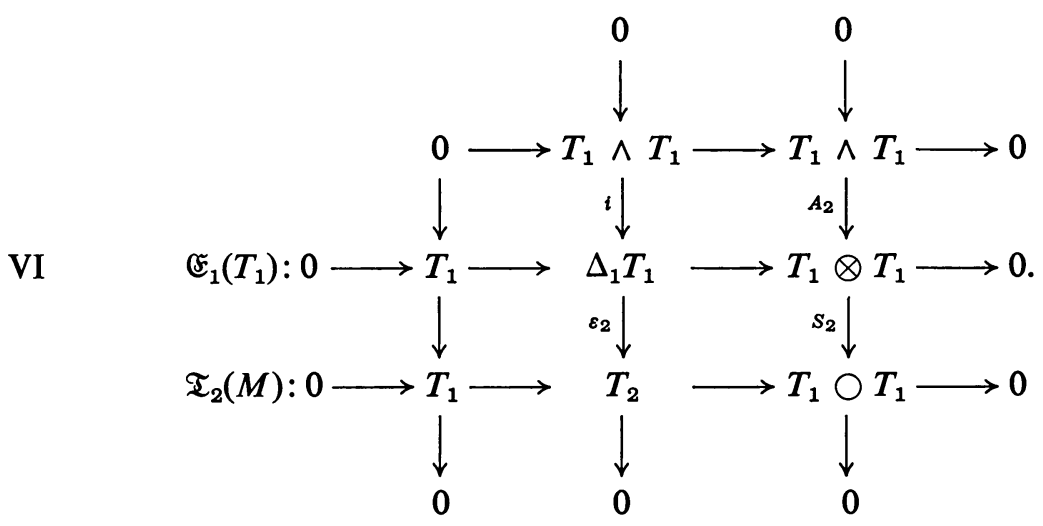

Proposition 5. If $\tilde{\omega}: \Delta_{1} T_{1} \rightarrow T_{1}$ is a connexion, the obstruction to splitting the bottom row in VI, which is a cross-section of $\operatorname{Hom}\left(T_{1} \wedge T_{1}, T_{1}\right)=T_{1}^{*} \wedge T_{1}^{*} \otimes T_{1}$, is the torsion tensor of $\tilde{\omega}$.

Proof. The kernel of $\varepsilon_{2}$ consists of all elements of the form

$$
q(-[E, F] \oplus E \otimes F-F \otimes E) .
$$

Hence $\tilde{\omega} i(E \wedge F)=\nabla_{E} F-\nabla_{F} E-[E, F]$, which is a standard formula for the torsion tensor [8, p. 133]. Q.E.D.

A connexion in $T_{1}$ is called symmetric if its torsion is zero. An arbitrary connexion in $T_{1}$ gives rise to a symmetric connexion called symmetrization of the given connexion, as may be seen in two ways. First, if $\Gamma_{j i}^{k}$ are the classical components of a connexion, the components of the symmetrization are $\frac{1}{2}\left(\Gamma_{j i}^{k}+\Gamma_{i j}^{k}\right)$. Alternatively, we observe that the right-hand column of VI splits naturally, so that a splitting of the middle row (i.e., a connexion in $T_{1}$ ) induces a splitting of the bottom row, by Lemma 2 . But the induced splitting of the bottom row induces, in turn, a splitting of the middle row with obstruction (i.e., torsion) zero, again by Lemma 2. This last connexion is the symmetrization of the given connexion. If $\nabla$ is the covariant derivative for a connexion in $T_{1}$, the covariant derivative for the symmetrized connexion is

$$
\nabla_{X}^{\prime} Y=\frac{1}{2}\left(\nabla_{X} Y+\nabla_{Y} X+[X, Y]\right) \text {. }
$$

Also we see that the splittings of $T_{2}(M)$ are in one-one correspondence with the symmetric connexions in $T_{1}$, which is a principal result of several previous publications [1], [4], [6]. 
We shall say that a construction based on a connexion is independent of the torsion if it remains unchanged when the given connexion is replaced by its symmetrization. An example is the induced splitting of $T_{2}(M)$ discussed in the previous paragraph.

As we have already observed, a connexion in a bundle induces a connexion in any algebraically associated bundle. For the case of the tensor product the covariant derivative for the induced connexion takes the form

$$
\nabla_{Y}\left(X_{1} \otimes X_{2}\right)=\left(\nabla_{Y} X_{1}\right) \otimes X_{2}+X_{1} \otimes \nabla_{Y} X_{2}
$$

It follows that the covariant derivative for the induced connexion in the symmetric product takes the form $\nabla_{Y}\left(X_{1} \bigcirc X_{2}\right)=\left(\nabla_{Y} X_{1}\right) \bigcirc X_{2}+X_{1} \bigcirc \nabla_{Y} X_{2}$.

If $\xi_{1}$ is a connexion in $T_{1}$ we shall denote the induced connexion in $\bigcirc^{p} T_{1}$ by $\xi_{1}^{p}$. This induced connexion has a certain symmetry property. Let $\nabla, \nabla^{\prime}$ be the covariant derivatives for $\xi_{1}^{p}$ and $\xi_{1}^{p^{\prime}}$, respectively, where $\xi_{1}^{p^{\prime}}$ is the connexion in $\bigcirc^{p} T_{1}$ induced by the symmetrization of $\xi_{1}$. Then

$$
\sum_{\sigma} \nabla_{X_{\sigma(1)}}^{\prime}\left(X_{\sigma(2)} \bigcirc \cdots \bigcirc X_{\sigma(p)}\right)=\sum_{\sigma} \nabla_{X_{\sigma(1)}}\left(X_{\sigma(2)} \bigcirc \cdots \bigcirc X_{\sigma(p)}\right),
$$

where the sum extends over all permutations $\sigma$ of $\{1, \ldots, p\}$. To prove this we observe that $\sum_{\sigma} \nabla_{X_{\sigma(1)}}^{\prime}\left(X_{\sigma(2)} \bigcirc \cdots \bigcirc X_{\sigma(p)}\right)$ is the sum of two expressions, namely

$\frac{1}{2} \sum_{\sigma} \sum_{i} X_{\sigma(2)} \bigcirc \cdots \bigcirc \nabla_{X_{\sigma(1)}}\left(X_{\sigma(i)}\right) \bigcirc X_{\sigma(i+1)} \bigcirc \cdots \bigcirc X_{\sigma(p)}$

and

$$
+X_{\sigma(2)} \bigcirc \cdots \bigcirc \nabla_{X_{\sigma(i)}}\left(X_{\sigma(1)}\right) \bigcirc \cdots \bigcirc X_{\sigma(p)}
$$

$$
\frac{1}{2} \sum_{\sigma} \sum_{i} X_{\sigma(2)} \bigcirc \cdots \bigcirc\left[X_{\sigma(1)}, X_{\sigma(i)}\right] \bigcirc \cdots \bigcirc X_{\sigma(p)}
$$

The first term is just $\sum_{\sigma} \nabla_{X_{\sigma(1)}}\left(X_{\sigma(2)} \bigcirc \cdots \bigcirc X_{\sigma(p)}\right)$, as we can see by changing the order of summation and then permuting 1 and $i$ in the second summand. The second term is both symmetric and anti-symmetric in its indices, so it vanishes.

Let $T_{p}(M)$ be as before, $p>1$, and define $T_{0}=0$ and $\mathfrak{I}_{1}(M): 0 \rightarrow 0 \rightarrow T_{1} \rightarrow T_{1} \rightarrow 0$.

THEOREM 6. An affine connexion $\xi_{1}$ in $M$ induces a unique connexion $\xi_{p}: \Delta_{1} T_{p} \rightarrow T_{p}$ in $T_{p}$ and a unique splitting $o_{p}: T_{p} \rightarrow T_{p-1}$ of $T_{p}(M)$ for each $p>0$ satisfying the conditions

$$
\begin{aligned}
\xi_{p}= & \xi_{p-1} \Delta_{1} o_{p}+\tilde{o}_{p} \xi_{1}^{p} \Delta_{1} P_{p} \\
o_{p}\left(X_{1} X_{2} \cdots X_{p}\right)= & X_{1} X_{2} \cdots X_{p}-\frac{1}{p !} \sum_{\sigma} X_{\sigma(1)} \cdots X_{\sigma(p)} \\
& +\frac{1}{p !} \sum_{\sigma} \tilde{o}_{p-1} \nabla_{X_{\sigma(1)}}\left(X_{\sigma(2)} \bigcirc \cdots \bigcirc X_{\sigma(p)}\right) \\
& +\frac{1}{p !} \sum_{\sigma} X_{\sigma(1)} o_{p-1}\left(X_{\sigma(2)} \cdots X_{\sigma(p)}\right)
\end{aligned}
$$


where the $X_{i}$ are vector fields, where the sums extend over all permutations $\sigma$, and $\tilde{o}_{p}$ is the map associated to $o_{p}$.

Proof. Let $o_{1}=0$. The uniqueness statements follow by an induction from (1) and (2).

To prove existence we proceed by recursion. The splittings $o_{1}$ and $\xi_{1}$ are already defined. Assume we have already defined $\xi_{p-1}$ and $o_{p-1}$. We have (by DGHO, Proposition 3.20) the commutative diagram with exact rows and columns

VII

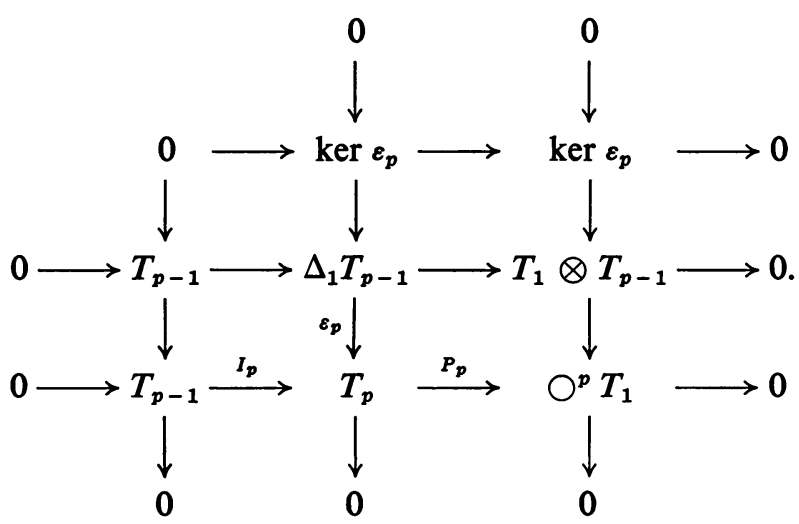

Then $o_{p-1}$ induces a splitting $\rho: \bigcirc^{p} T_{1} \rightarrow \otimes^{p} T_{1} \rightarrow T_{1} \otimes T_{p-1}$ of the right-hand column of VII, and this, together with $\xi_{p-1}$, the given splitting of the middle row, induces a splitting $o_{p}$ of the bottom row, by Lemma 2. This splitting induces an isomorphism $T_{p} \cong T_{p-1} \oplus \bigcirc^{p} T_{1}$, so that $\xi_{p-1}$ and $\xi_{1}^{p}$ induce a connexion $\xi_{p}$ in $T_{p}$, whose analytic expression is just (1).

By Lemma $2 o_{p}\left(X_{1} \cdots X_{p}\right)=X_{1} \cdots X_{p}-\varepsilon_{p} \xi_{p-1} \rho P_{p}\left(X_{1} \cdots X_{p}\right)$, where $\xi_{p-1}$ is the map associated to $\xi_{p-1}$. But this is, by a simple computation,

$$
\begin{aligned}
o_{p}\left(X_{1} \cdots X_{p}\right)= & X_{1} \cdots X_{p}-\frac{1}{p !} \sum_{\sigma} X_{\sigma(1)} \cdots X_{\sigma(p)} \\
& +\frac{1}{p !} \sum_{\sigma} X_{\sigma(1)} o_{p-1}\left(X_{\sigma(2)} \cdots X_{\sigma(p)}\right) \\
& +\frac{1}{p !} \sum_{\sigma} \nabla_{X_{\sigma(1)}} \tilde{o}_{p-1}\left(X_{\sigma(2)} \bigcirc \cdots \bigcirc X_{\sigma(p)}\right) .
\end{aligned}
$$

But it follows from condition (1) that

$$
\nabla_{X_{\sigma(1)} \tilde{o}_{p-1}}\left(X_{\sigma(2)} \bigcirc \cdots \bigcirc X_{\sigma(p)}\right)=\tilde{o}_{p-1} \nabla_{X_{\sigma(1)}}\left(X_{\sigma(2)} \bigcirc \cdots \bigcirc X_{\sigma(p)}\right)
$$

Condition (2) now follows and this completes the proof. 
THEOREM 7. An affine connexion $\xi_{1}$ in a real differentiable manifold $M$ induces a splitting $o_{p}: T_{p} \rightarrow T_{p-1}$ of the exact sequence

$$
\mathfrak{T}_{p}(M): 0 \rightarrow T_{p-1} \rightarrow T_{p} \rightarrow \bigcirc^{p} T_{1} \rightarrow 0
$$

for each $p$, which is independent of the torsion and is uniquely characterized by the condition that if $x_{1}, \ldots, x_{n}$ are affine normal coordinates centered at a point $x \in M$, then $o_{p}\left(\partial^{p} / \partial x_{i_{1}} \ldots \partial x_{i_{p}}\right)=0$, for all $i_{1}, \ldots, i_{p}$.

Proof. Since the fibre of $T_{p}$ at $x$ is spanned by $T_{p-1}$ together with all operators of the form $\partial^{p} / \partial x_{i_{1}} \cdots \partial x_{i_{p}}$ (DGHO, Lemma, p. 178), the splittings $o_{p}$ are uniquely characterized by the conditions of the theorem.

To prove the existence, we show that the splittings $o_{p}$ in Theorem 6 satisfy the conditions of the present theorem. First we show independence of the torsion by induction on $p$. For $p=1, o_{1}=0$ and there is nothing to show. Assume that $o_{p-1}$ and hence $\tilde{o}_{p-1}$ are independent of the torsion. Then in condition (2) of Theorem 6 , we see that the first, second, and fourth terms on the right are clearly independent of the torsion, and the third term on the right is independent of the torsion by the symmetry property of the induced connexion in $\bigcirc^{p} T_{1}$ previously established. Hence $o_{p}$ is independent of the torsion, as asserted.

Let $C(t)$ be a geodesic in $M$. We claim that $o_{p}\left(d^{p} / d t^{p}\right)=0$ on $C$. Again, we prove this by induction on $p$. For $p=1$ there is nothing to prove. Assume that $o_{p-1}\left(d^{p-1} / d t^{p-1}\right)=0$ on $C$. Then extend $d / d t$ to a local vector field on $M$ and apply condition (2) of Theorem 6. The first and second terms on the right cancel one another, and the third is zero on $C$ since $d / d t$ is by definition parallel along $C$ and hence so is $d / d t \bigcirc \cdots \bigcirc d / d t$. The fourth term is zero, since $o_{p-1}\left(d^{p-1} / d t^{p-1}\right)=0$ on $C$. Hence $o_{p}\left(d^{p} / d t^{p}\right)=0$ on $C$, as asserted.

Now let $x_{1}, \ldots, x_{n}$ be affine normal coordinates on $M$ centered at $x$. The condition defining such coordinates is that the curves $x_{1}=a_{1} t, \ldots, x_{n}=a_{n} t$ be geodesics, where the $a_{i}$ are constants. For these curves

$$
d^{p} / d t^{p}=\sum a_{i_{1}} \cdots a_{i_{p}} \partial^{p} / \partial x_{i_{1}} \cdots \partial x_{i_{p}} .
$$

The coefficients $a_{i_{1}} \cdots a_{i_{p}}\left(i_{1} \leqq \cdots \leqq i_{p}\right)$ satisfy no linear relation (DGHO, p. 178), and hence for each sequence $i_{1}, \ldots, i_{p}$, there are geodesics $C_{\alpha}\left(t_{\alpha}\right)$ through $x$ such that

$$
\partial^{p} / \partial x_{i_{1}} \cdots \partial x_{i_{p}}=\sum_{i} b_{i} d^{p} / d t_{\alpha}^{p}
$$

But $o_{p}\left(d^{p} / d t_{\alpha}^{p}\right)=0$. Since $o_{p}$ is a vector-bundle homomorphism,

$$
o_{p}\left(\partial^{p} / \partial x_{i_{1}} \cdots \partial x_{i_{p}}\right)=0
$$

and this completes the proof. 
Related to our characterization of the splittings $o_{p}$ is a property of the induced connexions $\xi_{p}$ of Theorem 6. Namely, if $C(t)$ is a geodesic, then $d^{p} / d t^{p}$ is parallel along $C$. To prove this we observe that condition (1) of Theorem 6 implies that

$$
\nabla_{t}\left(d^{p} / d t^{p}\right)=\nabla_{t} o_{p}\left(d^{p} / d t^{p}\right)+\tilde{o}_{p} \nabla_{t}(d / d t \bigcirc \cdots \bigcirc d / d t),
$$

where we have written $\nabla_{t}$ for the covariant derivative along $C$. The first term on the right vanishes, since $o_{p}\left(d^{p} / d t^{p}\right)=0$ on $C$, and the second vanishes by the parallelism of $d / d t$.

5. We shall next define and study the osculating spaces to a submanifold of an affinely or projectively connected manifold.

Definition. Let $f(t)$ be a curve in an affinely connected manifold $M$. Let $x_{1}, \ldots, x_{n}$ be affine normal coordinates centered at $f\left(t_{0}\right)$. The osculating space of order $p$ to $f$ at $t_{0}$ is the linear span, in the tangent space to $M$ at $f\left(t_{0}\right)$, of $\sum x_{i}^{\prime} \partial / \partial x_{i}$, $\sum x_{i}^{\prime \prime} \partial / \partial x_{i}, \ldots, \sum x_{i}^{(p)} \partial / \partial x_{i}$.

It is not hard to show that this definition is independent of the choice of affine normal coordinates and of the parametrization. Note that the osculating space of order one is just the tangent space, and that the condition for a curve to be a geodesic is that its osculating space of order $p$ coincide with its tangent space at each point.

DEFINITION. Let $f: K \rightarrow M$ be a differentiable mapping of a differentiable manifold $K$ into an affinely connected manifold $M$. The osculating space of order $p$ to $f$ at $t_{0} \in K$ is the linear span of the osculating spaces of order $p$ to curves on $K$ through $t_{0}$.

These definitions agree with those given in DGHO for ordinary affine spaces. Let $o_{p}$ be the splitting determined by Theorem 6 , and let

$$
\tilde{\omega}_{p}=o_{p} o_{p-1} \cdots o_{2}: T_{p}(M) \rightarrow T_{1}(M) .
$$

At the center of an affine normal coordinate system this map agrees with the map $\tilde{\omega}_{p}$ introduced in DGHO. Generalizing Proposition 2.3 (DGHO, p. 178) we have

THEOREM 8 (UNIVERSAL OSCULATION PROPERTY). Let $f: K \rightarrow M$ be a map of $a$ real differentiable manifold $K$ into an affinely connected manifold $M$. Then $\tilde{\omega}_{p} d^{p} f: T_{p}(K) \rightarrow T_{1}(M)$ maps onto the osculating spaces of order $p$, where $d^{p} f$ is the pth differential of $f(\mathrm{DGHO}, p .177)$.

Definition. By an affine degeneracy of order $p$ of a map $f: K \rightarrow M$ of a manifold $K$ into a space with linear connexion $M$, we mean a point of $K$ at which the rank of $\tilde{\omega}_{p} d^{p} f$ is less than the minimum of the fibre dimensions of $T_{p}(K)$ and $T_{1}(M)$.

Let us now give the projective generalization of these ideas. For the theory of projective connexions we refer to Kobayashi and Nagano [7]. Two symmetric 
affine connexions are said to be projectively related if they induce the same projective connexion. It is shown that two such connexions with components $\Gamma_{i j}^{k}$, $' \Gamma_{i j}^{k}$, respectively, are projectively related if and only if there is a linear differential form with components $\phi_{i}$ such that

$$
' \Gamma_{i j}^{k}=\Gamma_{i j}^{k}+\delta_{i}^{k} \phi_{j}+\delta_{j}^{k} \phi_{i} .
$$

Two symmetric affine connexions are projectively related if and only if they determine the same geodesics up to changes of parametrization.

Proposition 9. Any projective connexion on a paracompact manifold $M$ arises from a symmetric affine connexion.

Proof. Locally this is an easy consequence of statements in [7]. (Principal bundles $P^{2}(M) / G^{1}(n) \rightarrow M$ and $P^{2}(M) / H^{2}(n) \rightarrow M$ and a natural mapping

$$
\psi: P^{2}(M) / G^{1}(n) \rightarrow P^{2}(M) / H^{2}(n)
$$

are constructed such that the diagram

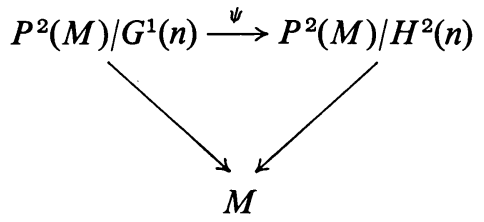

is commutative. Cross-sections of $P^{2}(M) / G^{1}(n) \rightarrow M$ are in one-one correspondence with symmetric affine connexions, and cross-sections of $P^{2}(M) / H^{2}(n) \rightarrow M$ are in one-one correspondence with projective connexions. If $\Gamma$ is a cross-section corresponding to an affine connexion, then $\psi \Gamma$ corresponds to the induced projective connexion. Now $\psi: P^{2}(M) / G^{1}(n) \rightarrow P^{2}(M) / H^{2}(n)$ is a principal bundle, and it has a local cross-section $\theta$. If $P$ is a cross-section corresponding to a projective connexion, $\theta P$ corresponds to an affine connexion inducing $P$.)

The proposition in its global form can now be proved easily using sheaf theory. Suppose $M$ has a projective connexion $P$. Let $\{U, V, \ldots\}$ be an open cover of $M$ by coordinate balls and choose a symmetric affine connexion ${ }_{U} \Gamma_{i j}^{k}$ in each $U$ which induces $P$ in $U$. In each $U \cap V,{ }_{U} \Gamma_{i j}^{k}$ and ${ }_{V} \Gamma_{i j}^{k}$ are projectively related. Hence there is a one-form $\phi_{i}^{U V}$ in $U \cap V$ such that ${ }_{V} \Gamma_{i j}^{k}-{ }_{U} \Gamma_{i j}^{k}=\delta_{i}^{k} \phi_{j}^{U V}+\delta_{j}^{k} \phi_{i}^{U V}$. This defines a 1-cocycle on $M$ with coefficients in $\mathscr{S}$, the sheaf of germs of differentiable tensor fields of type $(1,2)$ of the form $\delta_{i}^{k} \phi_{j}+\delta_{j}^{k} \phi_{i}$. Now the sum of two such tensor fields is again such, and so is a scalar multiple. Hence $\mathscr{S}$ is the sheaf of germs of sections of a vector bundle. By a well-known theorem of sheaf theory $H^{1}(M ; \mathscr{S})=0$. Hence $\delta_{i}^{k} \phi_{j}^{U V}+\delta_{j}^{k} \phi_{i}^{U V}$ is a coboundary, which implies that we can assign to each $U$ a one-form $\phi_{i}^{U}$ such that $\delta_{i}^{k} \phi_{j}^{V}+\delta_{j}^{k} \phi_{i}^{V}-\left(\delta_{i}^{k} \phi_{j}^{U}+\delta_{j}^{k} \phi_{i}^{U}\right)=\delta_{i}^{k} \phi_{j}^{U V}+\delta_{j}^{k} \phi_{i}^{U V}$. The connexion with components ${ }_{U} \Gamma_{i j}^{k}-\left(\delta_{i}^{k} \phi_{j}^{U}+\delta_{j}^{k} \phi_{i}^{U}\right)$ is projectively related to ${ }_{U} \Gamma_{i j}^{k}$ and agrees with 
${ }_{V} \Gamma_{i j}^{k}-\left(\delta_{i}^{k} \phi_{j}^{V}+\delta_{j}^{k} \phi_{i}^{V}\right)$ in $U \cap V$. This determines a symmetric affine connexion in $M$ globally which induces $P$. Q.E.D.

Proposition 10. Let $M$ be a manifold with two symmetric affine connexions, $\xi$ and $\xi^{\prime}$, which are projectively related. Then if $f: K \rightarrow M$ is a differentiable map, the osculating spaces of order $p$ to $f$ with respect to $\xi$ are the same as those with respect to $\xi^{\prime}$.

Proof. Since osculating spaces are spanned by osculating spaces to curves, it suffices to prove the proposition for curves. We shall make use of Theorem 8 , and so we begin by studying the splittings $o_{p}$ and $o_{p}^{\prime}$ induced by $\xi$ and $\xi^{\prime}$ respectively (Theorem 7). Let $\tilde{\omega}_{p}=o_{p} \cdots o_{2}$ and $\tilde{\omega}_{p}^{\prime}=o_{p}^{\prime} \cdots o_{2}^{\prime}$.

Let $C$ be a path in the sense of either connexion and let $t, s$ be affine parameters on $C$ with respect to $\xi$, $\xi^{\prime}$, respectively. Then $\tilde{\omega}_{p}\left(d^{r} / d t^{r}\right)=\tilde{\omega}_{p}^{\prime}\left(d^{r} / d s^{r}\right)=0$ on $C$, by the proof of Theorem 7 , for $1<r \leqq p$. But

$$
\frac{d^{p}}{d t^{p}}=\frac{d^{p} s}{d t^{p}} \frac{d}{d s}+\sum_{r=2}^{p} a_{r} \frac{d^{r}}{d s^{r}}
$$

for some functions $a_{r}$, by the chain rule. Hence

$$
\tilde{\omega}_{p}^{\prime}\left(d^{p} / d t^{p}\right)=\left(d^{p} s / d t^{p}\right)(d / d s)=\left(d^{p} s / d t^{p}\right)(d t / d s)(d / d t)
$$

which is a multiple of the tangent vector of $C$.

Now let $x_{1}, \ldots, x_{n}$ be affine normal coordinates in the sense of $\xi$ and centered at $x \in M$. As we saw in the proof of Theorem $7, \partial^{r} / \partial x_{i_{1}} \cdots \partial x_{i_{r}}$ can be written as a linear combination of operators $d^{p} / d t_{\alpha}^{p}$, where $C_{\alpha}\left(t_{\alpha}\right)$ are geodesics (in the sense of $\xi)$ through $x$. This is an algebraic result, and by considering a suitable subspace we find that these geodesics can be so chosen that their tangent vectors are linear combinations of $\partial / \partial x_{k}, k \in\left\{i_{1}, \ldots, i_{r}\right\}$. It follows that $\tilde{\omega}_{p}^{\prime}\left(\partial^{r} / \partial x_{i_{1}} \ldots \partial x_{i_{r}}\right)$ is a linear combination of $\partial / \partial x_{k}, k \in\left\{i_{1}, \ldots, i_{r}\right\}$.

Let $f(t)$ be a curve on $M$ through $x$. We shall prove the proposition by induction on $p$. Since the tangent line is defined without respect to a connexion, there is nothing to prove for $p=1$. Assume the proposition for $p-1$. Then let $q$ be the dimension of the osculating space of order $p-1$ at $x$, and by making an affine change of coordinates if necessary, assume that this space is spanned by $\partial / \partial x_{1}, \ldots$, $\partial / \partial x_{q}$ at $x$. It follows from the definition that in these new coordinates

$$
x_{i}^{\prime}=x_{i}^{\prime \prime}=\cdots=x_{i}^{(p-1)}=0,
$$

for $i>q$. Now $d^{p} / d t^{p}$ is the sum of $\sum x_{i}^{(p)} \partial / \partial x_{i}$ and terms of the form

$$
x_{i_{1}}^{\left(j_{1}\right)} \cdots x_{i_{r}}^{\left(j_{r}\right)} \frac{\partial^{r}}{\partial x_{i_{1}} \cdots \partial x_{i_{r}}}, \quad 0<j_{\beta}<p, \quad 1<r \leqq p,
$$


and these last vanish unless $i_{1}, \ldots, i_{r} \leqq q$. Hence

$$
\tilde{\omega}_{p}^{\prime}\left(d^{p} / d t^{p}\right) \equiv \sum x_{i}^{(p)} \partial / \partial x_{i}\left(\bmod \partial / \partial x_{1}, \ldots, \partial / \partial x_{q}\right)
$$

and hence

$$
\sum x_{i}^{(p)} \partial / \partial x_{i}=\tilde{\omega}_{p}\left(d^{p} / d t^{p}\right) \equiv \tilde{\omega}_{p}^{\prime}\left(d^{p} / d t^{p}\right)\left(\bmod \partial / \partial x_{1}, \ldots, \partial / \partial x_{q}\right),
$$

and by Theorem 8 , the osculating spaces of order $p$ agree at $x$. This completes the proof.

Let $M$ be a manifold with projective connexion $P$ and let $f: K \rightarrow M$ be a differentiable map.

Definition. By the osculating spaces of order $p$ to $f$ we mean the osculating spaces of order $p$ to $f$ with respect to some (and hence to any) symmetric affine connexion inducing $P$.

It is natural to ask under what circumstances affine and projective nondegenerate maps exist. Much progress on this problem has been made by E. A. Feldman [5] who has also given a number of examples of situations in which such maps do not exist. He has shown, in particular, that the real projective plane admits no immersion in $R^{10}$ without affine degeneracies of order three. We generalize this as follows.

Recall that a spin manifold is a differentiable manifold with vanishing second Stiefel-Whitney class.

THEOREM 11. Let $S$ be a spin manifold of dimension 10 provided with a projective connexion. Then the real projective plane admits no immersion in $S$ without projective degeneracies of order three.

Proof. Let $f: P^{2} \rightarrow S$ be a differentiable map. Provide $S$ with a symmetric affine connexion underlying the given projective connexion. If $f$ were free from affine degeneracies of order 3 , then $\tilde{\omega}_{3} d^{3} f: T_{3}\left(P^{2}\right) \rightarrow T_{1}(S)$ would be injective on each fibre. Since $T_{3}\left(P^{2}\right)$ has fibre dimension 9 , this would imply the existence of a line bundle $L$ such that $T_{3}\left(P^{2}\right) \oplus L \cong f^{!} T_{1}(S)$, where $f^{!}$denotes the inverse image of bundles. Now $T_{3}\left(P^{2}\right) \cong T_{1}\left(P^{2}\right) \oplus \bigcirc^{2} T_{1}\left(P^{2}\right) \oplus \bigcirc^{3} T_{1}\left(P^{2}\right)$. Recall that the StiefelWhitney class of $P^{2}$ is $1+\alpha+\alpha^{2}$, where $\alpha$ is the generator of $H^{1}\left(P^{2} ; Z_{2}\right)$. Let $w(L)=1+\delta$. Then, by an elementary computation using the product formula for Stiefel-Whitney classes and Proposition 3.24 of DGHO, we find that $w\left(T_{3}\left(P^{2}\right)\right)=$ $1+\alpha^{2}$ and hence $w\left(T_{3}\left(P^{2}\right) \oplus L\right)=1+\delta+\alpha^{2}$. But $w_{2}\left(f^{\prime} T_{1}(S)\right)=f^{*}\left(w_{2}\left(T_{1}(S)\right)\right)=0$, since $S$ is a spin manifold. Hence $T_{3}\left(P^{2}\right) \oplus L$ is not isomorphic to $f^{\prime} T_{1}(S)$, which is a contradiction. Q.E.D.

A noteworthy feature of this theorem is that though the notion of nondegeneracy depends on a connexion in $S$, the theorem is true for any connexion and depends only on the topological structure of $S$. 
We wish to mention, finally, that the theory of osculating spaces of submanifolds of affine spaces admits a generalization different from that studied above. We could define the osculating space of order $p$ to a curve in an affinely connected manifold to be the linear span of $X, \nabla_{X} X, \nabla_{X} \nabla_{X} X, \ldots, \nabla_{X}^{p-1} X$, where $X$ is the tangent field of the curve. Extend to higher-dimensional submanifolds as before. It is not hard to show that these osculating spaces depend only on the projective connexion derived from the given affine connexion. These osculating spaces agree with those studied above in orders 1 and 2, and in order 3 if the affine connexion is symmetric. It appears that the two theories diverge, in general, in order 4 and higher if the curvature does not vanish.

6. Appendix. H. Samelson and D. C. Spencer have indicated to us an alternative definition of $\Delta_{1} V$, as follows.

Atiyah [2] has defined an exact sequence

$$
0 \rightarrow \text { End } V \rightarrow Q \rightarrow T_{1} \rightarrow 0 .
$$

Tensoring with $V$ and letting $K=\operatorname{ker} \psi$, where $\psi:$ (End $V$ ) $\otimes V \rightarrow V$ is defined by $\psi(\phi \otimes v)=\phi(v)$, we get a commutative diagram with exact rows and columns

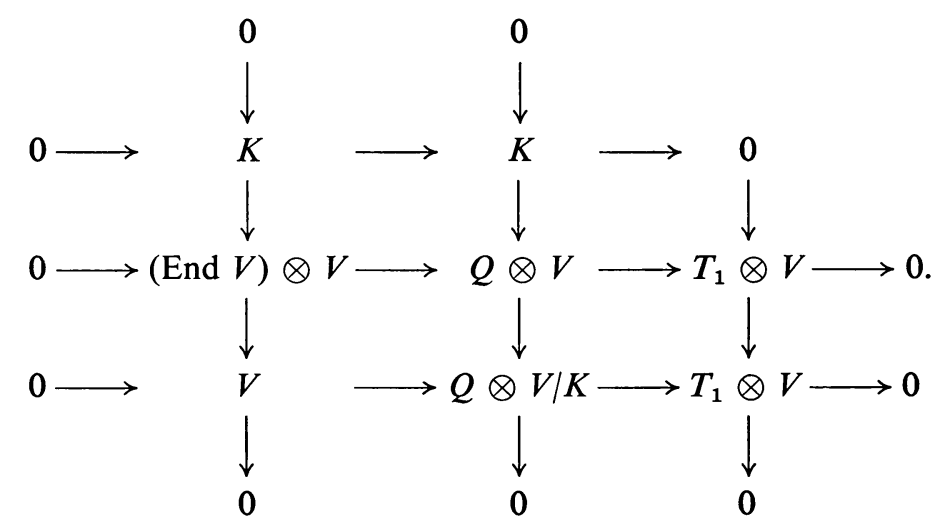

We may define $\Delta_{1} V=Q \otimes V / K$.

N. H. Kuiper had indicated another definition of $\Delta_{p} V$. Let $\Phi$ be the space of local functions which are linear on the fibres of $V$ and let $L^{p}$ be the space of $p$-jets of such functions at the zeros of $V$. Let $\left.\left(\Delta_{p} V\right)\right|_{x}=\operatorname{Hom}\left(\left.L^{p}\right|_{x}, F\right)$, where $F$ is the base field. In other words, let $\left.\left(\Delta_{p} \mathscr{V}\right)\right|_{x}$ be the subsheaf of $\operatorname{Hom}\left(\left.V^{*}\right|_{x}, F\right)$ consisting of operators of the form

$$
\sum S_{k}+\sum a_{i} \frac{\partial}{\partial x_{i}} S_{i}+\sum a_{i j} \frac{\partial^{2}}{\partial x_{i} \partial x_{j}} S_{i j}+\cdots+\sum a_{i_{1} \cdots i_{p}} \frac{\partial^{p}}{\partial x_{i_{1}} \cdots \partial x_{i_{p}}} S_{i_{1} \cdots i_{p}},
$$

where the $S$ 's represent sections of $V$. The functoriality, the exactness of the sequence $\mathfrak{E}_{p}(V)$, and the realization theorem follow quickly. 
Though the complications of defining $\Delta_{p} V$ by generators and relations as in DGHO may now be avoided, the relations worked out there between various bundles, as well as the analytic expressions, are still useful, as the present paper demonstrates.

The new definition appears to diverge from that of DGHO in the case of coherent sheaves, though at present we know of no application of the greater generality.

For another definition, as well as further discussion, the reader is referred to Libermann [9].

\section{REFERENCES} $163-178$.

1. W. Ambrose, R. S. Palais and I. M. Singer, Sprays, An. Acad. Brasil. Ci. 32 (1960),

2. M. F. Atiyah, Complex analytic connexions in fibre bundles, Trans. Amer. Math. Soc. 85 (1957), 181-207.

3. S. S. Chern, Complex manifolds, Notes of E. H. Spanier, Textos de Matematica, No. 5, Instituto de Fisica e Matematica, Universidade do Recife, 1959.

4. L. P. Eisenhart, Non-Riemannian geometry, Amer. Math. Soc. Colloq. Publ. Vol. 8, Amer. Math. Soc., Providence, R. I., 1927; reprint 1964.

5. E. A. Feldman, The geometry of immersions. I, Bull. Amer. Math. Soc. 69 (1963), 693-698; Trans. Amer. Math. Soc. 120 (1965), 185-224; II, Bull. Amer. Math. Soc. 70 (1964), 600-607.

6. S. Kobayashi, Canonical forms on frame bundles of higher-order contact, Differential Geometry, Proc. Sympos. Pure Math. Vol. 3, pp. 186-193, Amer. Math. Soc., Providence, R. I., 1961.

7. S. Kobayashi and T. Nagano, On projective connections, J. Math. Mech. 13 (1964), 215-235.

8. S. Kobayashi and K. Nomizu, Foundations of differential geometry. I, Interscience, New York, 1963.

9. P. Libermann, Sur la géométrie des prolongements des espaces fibrés vectoriels, Ann. Inst. Fourier (Grenoble) 14 (1964), 145-172.

10. W. F. Pohl, Differential geometry of higher order, Topology 1 (1962), 169-211. (Cited: DGHO.)

11. S. Sternberg, Lectures on differential geometry, Prentice-Hall, Englewood Cliffs, N. J., 1964; p. 103.

STANFORD UNIVERSITY,

StANFord, CALIFORNia 